\title{
Évaluation des doses délivrées au cours d'examens radiologiques
}

\section{PARAMĖTRES INTERVENANT DANS L'ÉVALUATION DES DOSES DÉLIVRÉES AU COURS D'UN EXAMEN RADIOLOGIQUE}

Les paramètres qui interviennent dans la dose délivrée au patient au cours d'un examen radiologique peuvent être répartis en deux principaux groupes: ceux liés à la technique d'examen ainsi que ceux liés aux caractéristiques et aux réglages de l'appareil.

Nous analysons l'influence de la variation de ces paramètres sur la dose délivrée au patient.

\subsection{La technique d'examen}

La dose délivrée au cours de la prise d'un cliché est évaluée en tenant compte des incidences, du format du film et de la tension compatible avec le contraste souhaité. Les conditions considérées se rapportent à des clichés dont la densité optique (d.o.) moyenne (1 à 1,5) est obtenue avec un couple film-écran standard.

Pour la tomographie, le temps de balayage dépend du type de coupe choisi mais la densité optique du film et la dose délivrée sont comparables à celles d'une incidence fixe.

La dose est également évaluée pour l'ensemble de l'examen c'est-à-dire en considérant le nombre total de clichés pratiqués ainsi que la durée de l'examen en scopie.

\subsection{Les paramètres liés aux caractéristiques et aux réglages de l'appareil}

\subsubsection{Le détecteur radiographique}

La dose délivrée au patient dépend du détecteur radiographique constitué de l'ensemble cassette couple film-écran.

- Matériau constituant la cassette

Par exemple, en utilisant une cassette en fibre de carbone, à une tension de $60 \mathrm{kV}$, la dose est réduite de $28 \%$ par rapport à une cassette en aluminium. A une tension plus élevée, $120 \mathrm{kV}$, la dose n'est réduite que de $18 \%[4-5]$.

VOL 23 - NUMERO SPÉCIAL. 


\section{- Ecrans}

Une réduction de la dose d'un facteur 2 à 4 est observée quand on utilise des écrans aux terres rares par rapport aux écrans standard. Cependant un couple film-écran mal adapté fait perdre le bénéfice d'une telle réduction de dose, et entraîne de plus une dégradation de la qualité de l'image [6-7].

\subsubsection{Le système de développement}

Un système de développement automatique est associé à pratiquement toutes les installations de radiodiagnostic.

Les conditions de développement des clichés agissent sur la qualité de l'image.

La température trop basse du révélateur, l'augmentation de la vitesse d'entraînement du film, la régénération du révélateur insuffisante, entraînent une diminution de la densité optique. Par contre, la variation en sens inverse de ces paramètres conduit à une augmentation de la densité optique.

On observe également une augmentation de la densité optique du voile du film lorsque le fixateur est mélangé au révélateur au cours d'une mauvaise manipulation. Par ailleurs, lorsque le révélateur est oxydé ou trop dilué, cela entraîne une diminution du contraste du cliché.

Le maintien de la qualité du système de développement doit permettre d'éviter de refaire un ou plusieurs clichés, et de délivrer des doses inutiles au patient.

\subsubsection{La tension}

Le choix de la plus haute tension compatible avec le contraste souhaité permet de réduire la dose délivrée au patient. En effet, le rapport de la dose entrée à la dose sortie du patient diminue lorsque la tension augmente.

Par exemple, pour une épaisseur de $20 \mathrm{~cm}$, lorsque la filtration est de $2,5 \mathrm{~mm}$ d'Al, une augmentation de la tension de $80 \mathrm{kV}$ à $100 \mathrm{kV}$ conduit à une réduction de la dose cutanée (à l'entrée du faisceau) de $35 \%$. A miépaisseur, cette réduction de la dose n'est que de $15 \%$.

\subsubsection{Les mAs}

La dose absorbée est directement proportionnelle au nombre de mAs. Ce paramètre est habituellement fixé manuellement ou automatiquement en fonction de la tension sélectionnée et de la distance foyer-film, de façon à assurer une densité optique optimale au niveau du film.

\subsubsection{Le générateur}

Le nombre de $\mathrm{mAs}$ indiqué dans les tableaux dosimétriques correspond à un générateur triphasé. Afin d'évaluer la dose délivrée au patient, le nombre de mAs doit être adapté au type de générateur utilisé.

Dans les mêmes conditions de géométrie, pour la même valeur de tension, la dose pour $1 \mathrm{mAs}$ délivrée par un générateur monophasé est 1,8 fois plus faible que celle fournie par un générateur triphasé. 


\subsubsection{La filtration}

La filtration permet d'éliminer les composantes de basse énergie qui ne peuvent atteindre le détecteur et qui irradient inutilement la surface cutanée. La filtration totale minimale doit être de $2 \mathrm{~mm}$ d'Al. Cependant, une filtration totale comprise entre $2,5 \mathrm{~mm}$ d'Al et $3,5 \mathrm{~mm}$ d'Al est souvent utilisée dans la gamme de tension comprise entre 70 et $100 \mathrm{kV}$.

A titre d'exemple pour une tension de $80 \mathrm{kV}$ et une filtration totale de $3 \mathrm{~mm}$ d'Al, la dose cutanée est $10 \%$ plus faible que celle obtenue avec une filtration de $2,5 \mathrm{~mm}$ d'Al [8].

\subsubsection{La grille antidiffusante}

La grille antidiffusante permet de réduire le rayonnement diffusé par le milieu examiné, améliorant ainsi la qualité de l'image.

Le facteur de Bucky est le facteur multiplicatif du nombre de mAs qui tient compte de l'atténuation par la grille du rayonnement parvenant au détecteur radiologique.

Une grille usuelle (de rapport 6) a été considérée: ce choix entraîne un facteur de Bucky de 4 à une tension moyenne de $80 \mathrm{kV}$ et cette valeur a été prise en compte pour l'estimation du nombre de mAs utilisé.

Par exemple, lorsqu'on utilise une grille dont le facteur de Bucky est égal à 5 , cela revient à augmenter le nombre de $\mathrm{mAs}$ dans le rapport $5 / 4=1,25$ (les autres paramètres étant bien sûr conservés) pour obtenir la même densité optique au niveau du film.

\subsubsection{La taille du champ d'irradiation}

Les conditions considérées supposent une stricte limitation du champ à la région d'intérêt sans recouvrir les organes adjacents, non concernés par l'examen. Afin de s'assurer que la dimension du champ a été correctement choisie, il est souhaitable qu'une bordure non irradiée autour du film soit visible.

\subsubsection{Les conditions de géométrie: distance foyer-film (DFF)}

A tension et mAs constants, la diminution de la DFF entraîne une augmentation de la dose au niveau du détecteur.

Afin de conserver la même densité optique au niveau du film (1 à 1,5) le nombre de mAs doit être corrigé, en première approximation, suivant la loi de l'inverse du carré des distances.

Par exemple, pour une même tension, lorsque deux expositions sont réalisées, l'une à DFF $110 \mathrm{~cm}$ et $100 \mathrm{mAs}$, l'autre à DFF $80 \mathrm{~cm}$, pour obtenir une densité optique voisine, le nombre de mAs doit être corrigé dans le rapport $(80 / 110)^{2}=0,53$. Le nombre de $m A$ s utilisé est alors $(100 \times 0,53)=53 \mathrm{mAs}$.

Par ailleurs, une diminution de la distance foyer-film entraîne une augmentation du rapport de la dose cutanée à la dose reçue par le film. La dose cutanée est donc plus élevée. 
A titre d'exemple, à une tension de $80 \mathrm{kV}$ et une épaisseur de patient de $20 \mathrm{~cm}$, lorsque la DFF est réduite de $110 \mathrm{~cm}$ à $80 \mathrm{~cm}$, cela entraîne une augmentation de $35 \%$ de la dose cutanée.

\subsubsection{La radioscopie télévisée}

Généralement, les examens comportent un temps de scopie. La tension utilisée et la durée de la scopie sont souvent notées, mais l'intensité du courant utilisé n'est pas toujours relevée.

L'évaluation dosimétrique de la phase de scopie de l'examen peut cependant être déduite des tableaux présentés, en considérant une valeur moyenne de l'intensité du courant égale à $2 \mathrm{~mA}$; le générateur fonctionne généralement en monophasé au cours de la scopie et c'est l'hypothèse qui a été retenue. Notons cependant qu'un très bon ensemble de scopie télévisée peut donner des images de qualité avec $0,5 \mathrm{~mA}$ ou qu'à l'inverse, une installation mal réglée comportant un amplificateur de luminance épuisé peut fonctionner avec un courant supérieur à $5 \mathrm{~mA}$.

\section{CALCUL DE LA DOSE dÉLIVRÉE AU NIVEAU DE LA RÉGION EXAMINÉE (POUR UN CLICHÉ)}

\subsection{Paramètres fixés pour le calcul}

- Le type de générateur (monophasé ou triphasé).

- La tension (kV).

- La distance foyer-film (DFF).

- Le nombre de mAs.

- La dimension du champ (format du film).

- L'épaisseur du patient.

\subsection{Eléments de calcul}

\subsection{1. "Dose dans l'air"}

La dose dans l'air $\left(D_{a}\right)$ correspond à la dose délivrée à un élément de tissu (eau), infiniment petit, isolé dans l'air, à une distance donnée de la source sur l'axe du faisceau pour un champ de $30 \times 30 \mathrm{~cm}^{2}$.

Pour $1 \mathrm{mAs}$ à $1 \mathrm{~m}$ du foyer, $\mathrm{D}_{\mathrm{a}}(\mathrm{mG} / \mathrm{mAs})$ peut être calculée en fonction de la tension $(U)$, de la filtration totale et du type de générateur par les relations données ci-dessous [9-10].

\begin{tabular}{|c|c|}
\hline $\begin{array}{l}\text { Filtration } \\
\text { totale } \\
\mathrm{mm} \mathrm{Al}\end{array}$ & $\begin{array}{c}\left(\mathrm{D}_{\mathrm{a}}\right) \text { : Dose dans l'air (mGy/mAs) } \\
\text { à } 100 \mathrm{~cm} \text { du foyer } \\
\text { pour un générateur triphasé }\end{array}$ \\
\hline 2 & $5,13 \quad 10^{-5} \quad U^{1,74}$ \\
\hline 2,5 & $4,30 \quad 10^{-5} \quad U^{1,74}$ \\
\hline 3 & $3,74 \quad 10^{-5} \quad U^{1,74}$ \\
\hline
\end{tabular}


Le tableau I fournit les valeurs calculées des $D_{a}$ *.

TABLEAU ।

DÉBIT DE DOSE ( $\mathrm{mGy} / \mathrm{mAs}$ ) DANS L'AIR EN FONCTION

DE LA TENSION ET DE LA FILTRATION A 100 CM DU FOYER POUR UN CHAMP DE $30 \mathrm{CM} \times 30 \mathrm{CM}^{* *}$

\begin{tabular}{|c|c|c|c|}
\hline \multirow{2}{*}{$k V$} & \multicolumn{3}{|c|}{ Filtration mm Al } \\
\cline { 2 - 4 } 50 & 2,0 & 2,5 & 3,0 \\
\hline 60 & 0,046 & 0,039 & 0,034 \\
70 & 0,064 & 0,053 & 0,046 \\
80 & 0,083 & 0,070 & 0,061 \\
90 & 0,105 & 0,088 & 0,077 \\
100 & 0,129 & 0,105 & 0,094 \\
110 & 0,155 & 0,130 & 0,113 \\
120 & 0,183 & 0,153 & 0,133 \\
130 & 0,213 & 0,176 & 0,155 \\
140 & 0,245 & 0,205 & 0,178 \\
150 & 0,278 & 0,233 & 0,203 \\
\hline
\end{tabular}

* Pour les générateurs monophasés $D_{a}$ monophasé $=\frac{\text { Da triphasé }}{1,8}$

** Pour un champ de $20 \times 20 \mathrm{~cm}^{2}: \mathrm{D}_{\mathrm{a}} \times 0,98$

Pour un champ de $10 \times 10 \mathrm{~cm}^{2}: \mathrm{D}_{\mathrm{a}} \times 0,92$

\subsubsection{Dose à la surface d'entrée du milieu}

La dose à la surface d'entrée $\left(D_{e}\right)$ du milieu (eau) est calculée en fonction de la distance foyer-surface d'entrée (DFE), de la tension (U), de la filtration totale $(F)$ et de la dimension du champ $\left(A_{e}\right)$ à la surface d'entrée, pour le nombre de mAs utilisé pour un cliché selon la formule ci-dessous.

$$
D_{e}=D_{a} \cdot\left(\frac{100}{D F E}\right)^{2} \cdot F^{2} D_{\left(A_{e}\right)} \cdot m A s
$$

avec $F R D_{\left(A_{e}\right)}$ : facteur de rétrodiffusion compte tenu de la taille du champ à la surface d'entrée [11-12] (tableau II). 
TABLEAU II (d'après 11-12)

FACTEURS DE RÉTRODIFFUSION (FRD)

(Champ $30 \mathrm{~cm} \times 30 \mathrm{~cm}$ )

\begin{tabular}{|c|c|}
\hline Tension $(\mathrm{kV})$ & FRD \\
\hline 50 & 1,25 \\
60 & 1,32 \\
70 & 1,35 \\
80 & 1,40 \\
90 & 1,42 \\
100 & 1,46 \\
110 & 1,48 \\
120 & 1,50 \\
130 & 1,52 \\
140 & 1,52 \\
\hline
\end{tabular}

\subsubsection{Dose en profondeur dans le milieu}

La Dose $\left(D_{z}\right)$ en un point du milieu situé à une profondeur $P_{z}$ est calculée en fonction de la distance du foyer au point considéré (DFZ), de la tension (U), de la filtration totale $(F)$, des dimensions du champ $\left(A_{z}\right)$ à la profondeur $\mathrm{P}_{\mathrm{z}}$ pour le nombre de $\mathrm{mAs}$ utilisé pour un cliché selon la formule ci-dessous.

$$
D_{z}=D_{a} \cdot\left(\frac{100}{D F Z}\right)^{2} \cdot \operatorname{RTA}_{\left(A_{z}, P_{z}\right)} \cdot m A s
$$

avec RTA $A_{\left(A_{z}, P_{z}\right)}$ : rapport tissu-air compte tenu de la taille du champ $A_{z}$ à la profondeur $P_{z}$. (Fig. 1,2 et 3 ).

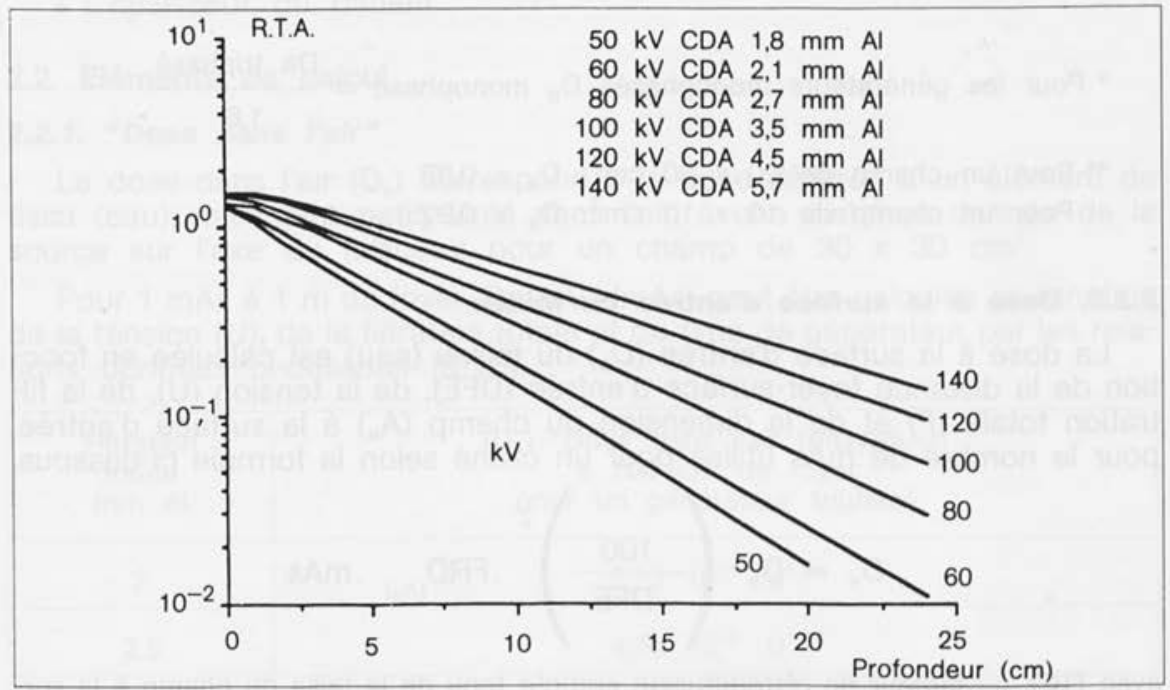

Fig. 1. - R.T.A. POUR DIFFÉRENTES QUALITÉS DE FAISCEAU (Champ $30 \mathrm{~cm} \times 30 \mathrm{~cm}$ - Filtration totale: $2,5 \mathrm{~mm} \mathrm{Al}$ ) 


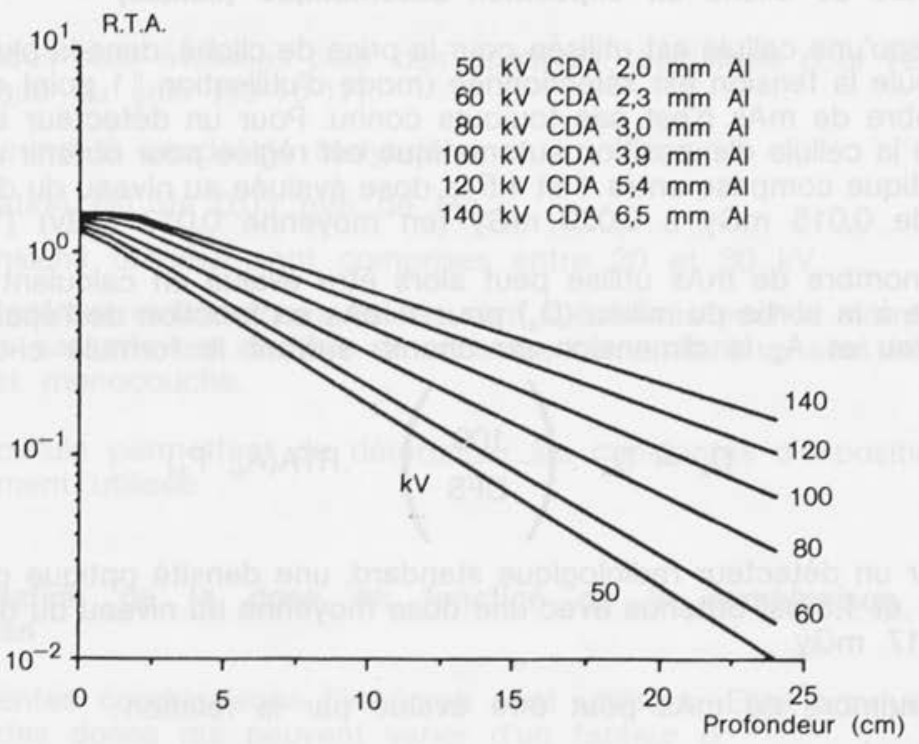

Fig. 2. - R.T.A. POUR DIFFÉRENTES QUALITÉS DE FAISCEAU (Champ $30 \mathrm{~cm} \times 30 \mathrm{~cm}$ - Filtration totale : $3 \mathrm{~mm}$ Al)

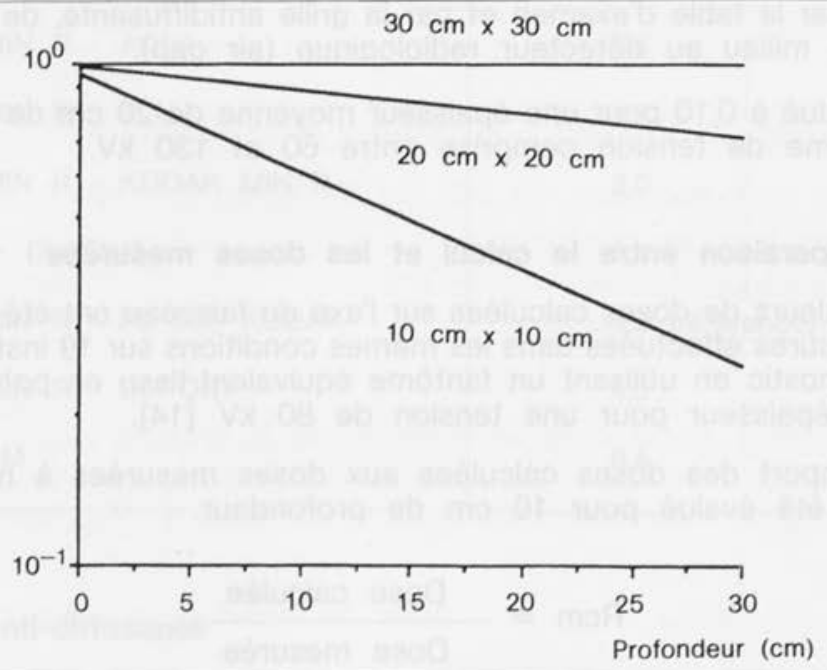

Fig. 3. - VARIATION RELATIVE DU R.T.A. EN FONCTION DE LA TAILLE DU CHAMP (Champ de référence : $30 \mathrm{~cm} \times 30 \mathrm{~cm}$ ) 


\subsection{Prise de cliché en exposition automatique (cellule)}

Lorsqu'une cellule est utilisée pour la prise de cliché, dans la plupart des cas, seule la tension est sélectionnée (mode d'utilisation "1 point cellule"); le nombre de mAs n'est pas toujours connu. Pour un détecteur standard, lorsque la cellule d'exposition automatique est réglée pour obtenir une densité optique comprise entre 1 et 1,5 la dose évaluée au niveau du détecteur varie de 0,015 mGy à 0,020 mGy (en moyenne 0,017 mGy) [13].

Le nombre de mAs utilisé peut alors être évalué en calculant la dose délivrée à la sortie du milieu $\left(D_{s}\right)$ pour $1 \mathrm{mAs}$ en fonction de l'épaisseur $P_{s}$ du milieu et $A_{s}$ la dimension du champ suivant la formule ci-dessous.

$$
D_{s}=D_{a} \cdot\left(\frac{100}{D F S}\right)^{2} \cdot \operatorname{RTA}\left(A_{s}, P_{s}\right)
$$

Pour un détecteur radiologique standard, une densité optique comprise entre 1 et 1,5 est obtenue avec une dose moyenne au niveau du détecteur de 0,017 mGy.

Le nombre de mAs peut être évalué par la relation:

$$
m A s=\frac{0,017}{D_{s} \cdot f_{c}}
$$

où $f_{c}$ tient compte du manque de rétro-diffusé à la sortie du milieu, de l'atténuation par la table d'examen et par la grille antidiffusante, de la distance sortie du milieu au détecteur radiologique (air gap).

$f_{c}$ est évalué à 0,10 pour une épaisseur moyenne de $20 \mathrm{~cm}$ de milieu dans une gamme de tension comprise entre 60 et $130 \mathrm{kV}$.

\subsection{Comparaison entre le calcul et les doses mesurées}

Les valeurs de doses calculées sur l'axe du faisceau ont été comparées à des mesures effectuées dans les mêmes conditions sur 19 installations de radiodiagnostic en utilisant un fantôme équivalent-tissu en polystyrène de $20 \mathrm{~cm}$ d'épaisseur pour une tension de $80 \mathrm{kV}$ [14].

Le rapport des doses calculées aux doses mesurées à mi-épaisseur $(R \mathrm{~cm})$ a été évalué pour $10 \mathrm{~cm}$ de profondeur.

$$
\mathrm{Rcm}=\frac{\text { Dose calculée }}{\text { Dose mesurée }}
$$

Le Rcm moyen est de 1,13 avec un écart type de 0,24 soit un écart type relatif de l'ordre de $20 \%$. 


\section{MAMMOGRAPHIE}

Les doses sont évaluées pour des appareils spécialisés pour l'examen radiologique du sein [15-16-17].

- le tube est à anode de Molybdène,

- la filtration est de 0,03 $\mathrm{mm}$ de Mo,

- les tensions utilisées sont comprises entre 20 et $30 \mathrm{kV}$,

- les détecteurs radiologiques utilisés sont spécialisés pour la mammographie: la cassette est en fibre de carbone, comportant un seul écran, le film est monocouche.

Une cellule permettant de déterminer les constantes d'exposition est généralement utilisée.

3.1. Variation de la dose en fonction de la combinaison film écran

Différentes combinaisons film-écran sont utilisées. Elles conduisent à délivrer des doses qui peuvent varier d'un facteur 6 .

\begin{tabular}{|l|l|}
\hline \multicolumn{1}{|c|}{ Couples film-écran } & $\begin{array}{l}\text { Rapport des doses } \\
\text { pour une d.o. }=1\end{array}$ \\
\hline KODAK MIN R - AGFA & 1,4 \\
KODAK MIN R - 3 M & 1,8 \\
KODAK MIN R - KODAK MIN R & 2,0 \\
DUPONT - DUPONT & 3,1 \\
KODAK MIN R - PE 205 KODAK & 1,0 (référence) \\
KODAK MIN R - DUPONT & 4,0 \\
3 M - 3 M & 6,4 \\
\hline
\end{tabular}

\subsection{Grille anti-diffusante} de 2.

Lorsqu'une grille antidiffusante est utilisée, le facteur de Bucky est VOL. 23 - NUMERO SPÉCIAL 
3.3. Variation de la dose - Conditions standard (faible agrandissement)

- Pour une épaisseur de $4,2 \mathrm{~cm}$ de milieu équivalent sein (plexiglas).

- Densité optique 1 au niveau du film.

- Avec grille antidiffusante.

3.3.1. Variation de la dose en fonction de la tension

\begin{tabular}{|l|c|c|c|c|}
\hline Tension (kV) & 22 & 25 & 27 & 30 \\
\hline Dose entrée (mGy) & 30 & 20 & 16 & 10 \\
\hline Dose mi-épaisseur (mGy) & 1,80 & 1,40 & 1,28 & 1,05 \\
\hline
\end{tabular}

3.3.2. Variation de la dose en fonction de l'épaisseur d'équivalent sein pour une tension de $27 \mathrm{kV}$

\begin{tabular}{|l|c|c|c|c|c|}
\hline Epaisseur (cm) & 2,8 & 3,2 & 3,7 & 4,2 & 5,1 \\
\hline Dose entrée (mGy) & 5,6 & 7,2 & 9,7 & 16,0 & 32,4 \\
\hline Dose mi-épaisseur (mGy) & 0,90 & 1,01 & 1,17 & 1,28 & 1,62 \\
\hline
\end{tabular}

3.4. Variation de la dose - Conditions d'agrandissement

- Pour une épaisseur de $4,2 \mathrm{~cm}$ de milieu équivalent sein.

- Densité optique 1.

- Agrandissement 1,5.

- Sans grille antidiffusante.

3.4.1. Variation de la dose en fonction de la tension

\begin{tabular}{|l|c|c|c|c|}
\hline Tension (kV) & 22 & 25 & 27 & 30 \\
\hline Dose entrée (mGy) & 83 & 60 & 45 & 28 \\
\hline Dose mi-épaisseur (mGy) & 5,0 & 4,2 & 3,6 & 2,8 \\
\hline
\end{tabular}


COMMISSION RADIODIAGNOSTIC - SFPH

3.4.2. Variation de la dose en fonction de l'épaisseur de milieu équivalent sein pour une tension de $27 \mathrm{kV}$

Agrandissement de 1,5

\begin{tabular}{|l|c|c|c|c|c|}
\hline Epaisseur (cm) & 2,8 & 3,2 & 3,7 & 4,2 & 5,1 \\
\hline Dose entrée (mGy) & 16,5 & 21,41 & 27,5 & 45,0 & 92,4 \\
\hline Dose mi-épaisseur (mGy) & 2,70 & 3,01 & 3,30 & 36,00 & 4,60 \\
\hline
\end{tabular}

N.B. Pour un agrandissement 1,5 la dose est augmentée d'un facteur 3 et pour un agrandissement de 2 de la dose est augmentée d'un facteur 4,5 par rapport aux conditions standard.

\section{SCANOGRAPHIE}

\subsection{Paramètres intervenant sur la dose délivrée au cours d'un examen scanographique}

En scanographie la dose est délivrée par un mince faisceau en éventail effectuant une rotation autour du patient.

La largeur à mi-hauteur du profil de la distribution de la dose du faisceau définit l'épaisseur de coupe correspondant à la zone irradiée.

La dose délivrée au cours d'un examen scanographique est exprimée soit pour une coupe unique, soit pour des coupes multiples jointives [18-19-20].

Pour une coupe unique, la dose à la surface est exprimée par la dose maximale correspondant au pic du profil de la distribution des doses dans le faisceau.

Pour des coupes multiples jointives, compte tenu de la forme du profil de la distribution des doses dans le faisceau, une portion de la dose est déposée au milieu d'une coupe adjacente. La dose en coupes multiples est donc plus élevée que dans le cas d'une coupe unique mais tend vers une valeur de saturation à partir de trois coupes jointives.

\subsubsection{Mouvements de rotation du tube}

Pour les scanographes de première génération dont la rotation du tube est de $180^{\circ}$, la dose au niveau de la peau est plus élevée du côté du tube qu'à la sortie.

VOL. 23 - NUMÉRO SPÉCIAL 
Les scanographes de $3^{e}$ et $4^{e}$ génération, dont la rotation du tube est de $360^{\circ}$, délivrent une dose plus uniforme à la surface de la peau qui décroît vers le centre de l'objet examiné.

\subsubsection{Tension et filtration}

Les valeurs de tension utilisées en scanographie sont comprises entre $120 \mathrm{kV}$ et $140 \mathrm{kV}$.

L'augmentation de la tension et de la filtration tend à homogénéiser la dose à l'intérieur, ainsi les écarts de doses entre la surface et le centre de la coupe sont réduits.

\subsection{3. $\mathrm{mAs}$}

La dose est proportionnelle aux mAs.

\subsubsection{Coupes multiples}

La dose délivrée au cours d'un examen en coupes multiples dépend de la distance entre deux coupes.

Le rapport :

$$
\text { Dose coupes multiples }
$$

$$
\text { Dose coupe unique }
$$

traduit l'effet de la zone de pénombre du profil de la dose en coupe unique sur une coupe adjacente.

En effet, par exemple, pour deux coupes jointives de $10 \mathrm{~mm}$ d'épaisseur ce rapport est égal à 1,5 .

Il dépend également de la largeur de la coupe: pour deux coupes fines jointives, il peut être égal ou supérieur à 3 .

Ce rapport tend vers une valeur de saturation à partir de trois coupes jointives.

II reste constant lorsque le nombre de coupes augmente.

\subsubsection{Taille de l'objet à examiner}

La dose à la surface augmente lorsque la taille de l'objet à examiner diminue.

L'optimisation des constantes radiologiques à la taille du sujet, par exemple la tension et le nombre de mAs (séquence particulière pour l'enfant) conduit à réduire la dose délivrée au cours de l'examen. 


\section{5. ÉVALUATION DES DOSES DÉlivRÉES POUR DIFFÉRENTS TYPES D'EXAMEN}

\subsection{Crâne}

5.2. Rachis cervical

5.3. Rachis dorsal

5.4. Rachis lombaire

5.5. Hystérographie

5.6. Thorax

5.7. Lavement baryté

5.8. Transit Gastro-Duodénal (TGD)

5.9. Rachis sacré

5.10. Bassin

5.11. Abdomen Sans Préparation (ASP)

5.12. Urographie Intra-veineuse (UIV)

5.13. Mammographie

5.14. Scanographie 


\section{CRANE}

\begin{tabular}{|c|c|c|c|c|c|}
\hline \multicolumn{2}{|c|}{$\begin{array}{l}\text { Distance foyer-film } \\
\text { Type de générateur } \\
\text { Filtration totale }\end{array}$} & \multicolumn{4}{|c|}{$\begin{array}{l}110 \mathrm{~cm} \\
\text { triphasé } \\
2,5 \mathrm{~mm} \mathrm{Al}\end{array}$} \\
\hline \multirow{2}{*}{ Incidences } & \multirow{2}{*}{$\begin{array}{c}\text { Tension } \\
\text { kV }\end{array}$} & \multirow[b]{2}{*}{$\mathrm{mAs}$} & \multirow{2}{*}{$\begin{array}{l}\text { Epaisseur de } \\
\text { la région examinée } \\
\text { en cm }\end{array}$} & \multicolumn{2}{|c|}{$\begin{array}{l}\text { Dose pour un cliché } \\
\text { en mGy ( } 100 \text { mrad) }\end{array}$} \\
\hline & & & & Entrée & Mi-épaisseur \\
\hline $\mathrm{AP}$ & 65 & 140 & 22 & 18,00 & 1,80 \\
\hline Profil & 60 & 50 & 15 & 4,70 & 0,90 \\
\hline Oblique & 60 & 90 & 18 & 9,20 & 1,30 \\
\hline Oblique & 65 & 95 & 20 & 12,00 & 1,50 \\
\hline
\end{tabular}

\section{ESTIMATION DE LA DOSE POUR L'ENSEMBLE D'UN EXAMEN TYPE}

- Pour un temps de scopie de 29 s

- Pour un nombre moyen de clichés de 2,9

\begin{tabular}{|c|c|c|}
\hline \multicolumn{3}{|c|}{ Doses pour l'ensemble de l'examen (mGy) } \\
\hline Mi-épaisseur & Cristallin & \\
\hline $4,50(0,60)^{\star}$ & 18,00 & \\
\hline
\end{tabular}

* Dose délivrée au cours du temps de scopie. 


\section{RACHIS CERVICAL}

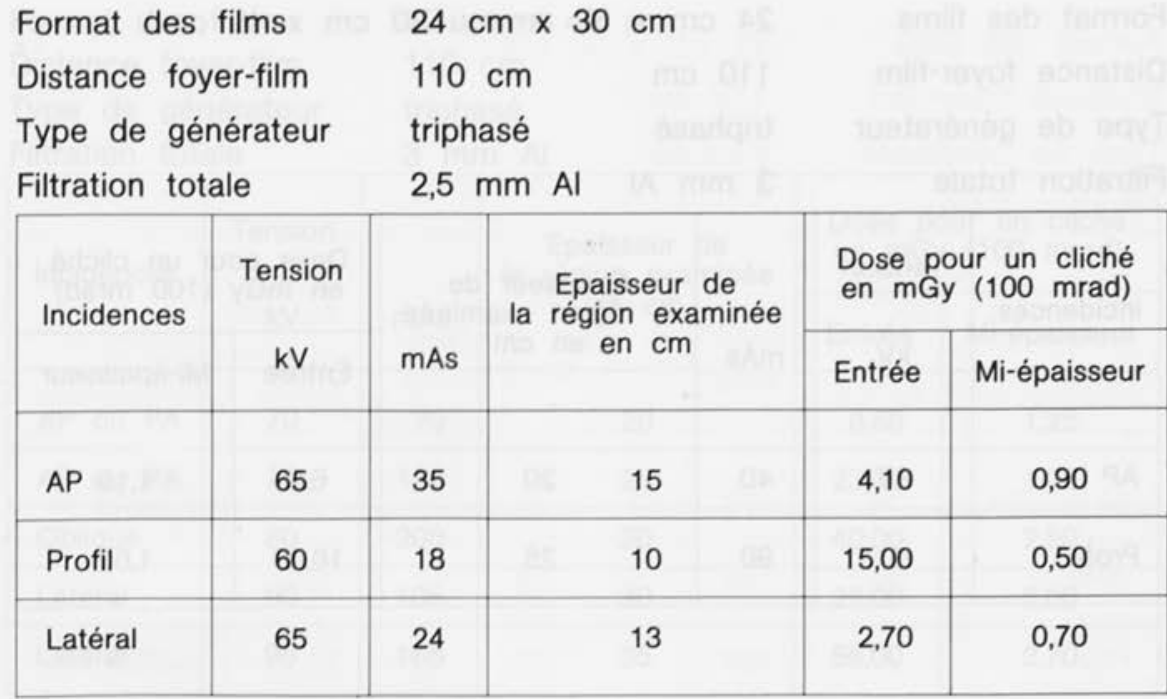

\section{ESTIMATION DE LA DOSE POUR L'ENSEMBLE D'UN EXAMEN TYPE}

- Pour un temps de scopie de 52 s

- Pour un nombre moyen de clichés de 3,6

\begin{tabular}{|l|l|l|}
\hline \multicolumn{2}{|c|}{ Doses pour l'ensemble de l'examen (mGy) } \\
\hline Mi-épaisseur & & \\
\hline $3,80(1,30)^{\star}$ & & \\
\hline
\end{tabular}

* Dose délivrée au cours du temps de scopie. 


\section{RACHIS DORSAL}

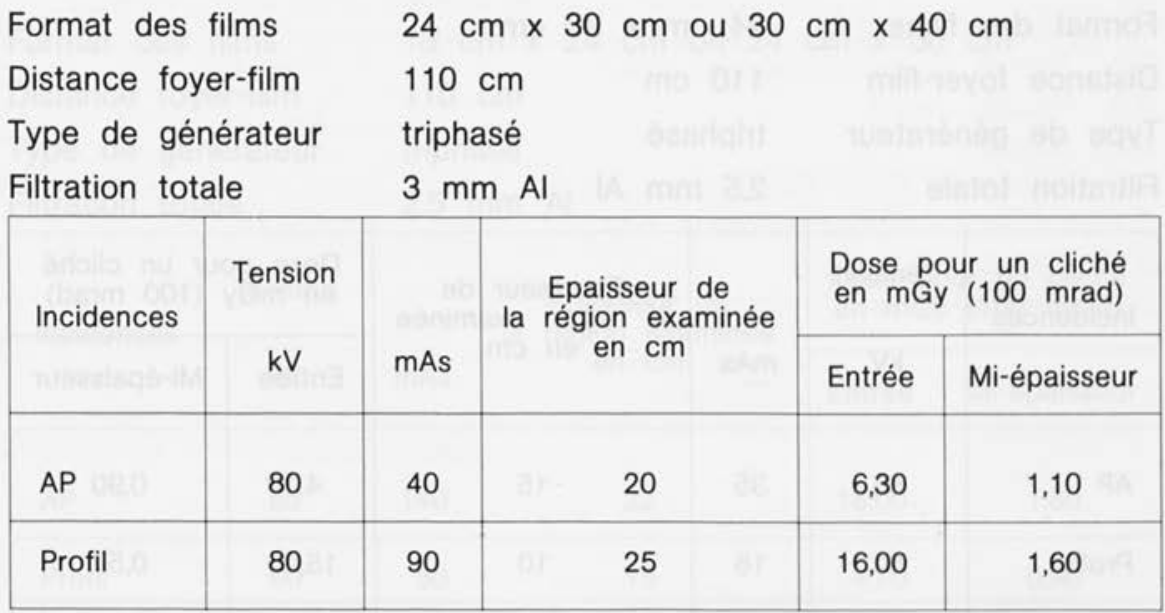

\section{ESTIMATION DE LA DOSE POUR L'ENSEMBLE D'UN EXAMEN TYPE}

- Pour un temps de scopie de 32 s

- Pour un nombre moyen de clichés de 3,7

\begin{tabular}{|c|c|c|}
\hline \multicolumn{2}{|c|}{ Doses pour l'ensemble de l'examen (mGy) } \\
\hline Corps vertébral - D7 & Ovaires & Testicules \\
\hline $8,00(0,55)^{*}$ & $<0,1$ & $<0,1$ \\
\hline
\end{tabular}

* Dose délivrée au cours du temps de scopie. 


\section{RACHIS LOMBAIRE}

Format des films

Distance foyer-film

Type de générateur

Filtration totale

\begin{tabular}{|c|c|c|c|c|c|}
\hline \multirow{2}{*}{ Incidences } & \multirow{2}{*}{$\begin{array}{l}\text { Tension } \\
\text { kV }\end{array}$} & \multirow[b]{2}{*}{ mAs } & \multirow{2}{*}{$\begin{array}{l}\text { Epaisseur de } \\
\text { la région examinée } \\
\text { en } \mathrm{cm}\end{array}$} & \multicolumn{2}{|c|}{$\begin{array}{l}\text { Dose pour un cliché } \\
\text { en mGy ( } 100 \text { mrad) }\end{array}$} \\
\hline & & & & Entrée & Mi-épaisseur \\
\hline$A P$ ou $P A$ & 70 & 70 & 20 & 8,60 & 1,25 \\
\hline AP ou PA & 70 & 165 & 25 & 23,00 & 1,95 \\
\hline Oblique & 80 & 200 & 30 & 40,00 & 2,50 \\
\hline Latéral & 90 & 105 & 30 & 28,00 & 2,00 \\
\hline Latéral & 95 & 165 & 35 & 56,00 & 2,70 \\
\hline
\end{tabular}

$30 \mathrm{~cm} \times 40 \mathrm{~cm}$

$110 \mathrm{~cm}$

triphasé

$3 \mathrm{~mm} \mathrm{Al}$ 


\section{HYSTEROGRAPHIE}

Format des films

Distance foyer-film

Type de générateur

Filtration totale

\begin{tabular}{|c|c|c|c|c|c|}
\hline \multirow{2}{*}{ Incidences } & \multirow{2}{*}{$\begin{array}{c}\text { Tension } \\
\text { kV }\end{array}$} & \multirow[b]{2}{*}{ mAs } & \multirow{2}{*}{$\begin{array}{l}\text { Epaisseur de } \\
\text { la région examinée } \\
\text { en } \mathrm{cm}\end{array}$} & \multicolumn{2}{|c|}{$\begin{array}{l}\text { Dose pour un cliché } \\
\text { en mGy (100 mrad) }\end{array}$} \\
\hline & & & & Entrée & Mi-épaisseur \\
\hline AP ou PA & 70 & 70 & 20 & 9,60 & 1,25 \\
\hline Oblique & 70 & 165 & 25 & 23,00 & 1,90 \\
\hline Profil & 70 & 400 & 30 & 63,00 & 3,00 \\
\hline
\end{tabular}

$24 \mathrm{~cm} \times 30 \mathrm{~cm}$

$110 \mathrm{~cm}$

triphasé

$3 \mathrm{~mm} \mathrm{Al}$

ESTIMATION DE LA DOSE POUR L'ENSEMBLE D'UN EXAMEN TYPE

- Pour un temps de scopie de $90 \mathrm{~s}$

- Pour un nombre moyen de clichés de 4,6

\begin{tabular}{|c|c|c|}
\hline \multicolumn{2}{|c|}{ Doses pour l'ensemble de l'examen (mGy) } \\
\hline Ovaires & & \\
\hline $10,50(1,00)^{*}$ & & \\
\hline
\end{tabular}

* Dose délivrée au cours du temps de scopie. 


\section{THORAX}

Format des films

$36 \mathrm{~cm} \times 43 \mathrm{~cm}$

Distance foyer-film

$110 \mathrm{~cm}$

Type de générateur triphasé

Filtration totale

$3 \mathrm{~mm} \mathrm{Al}$

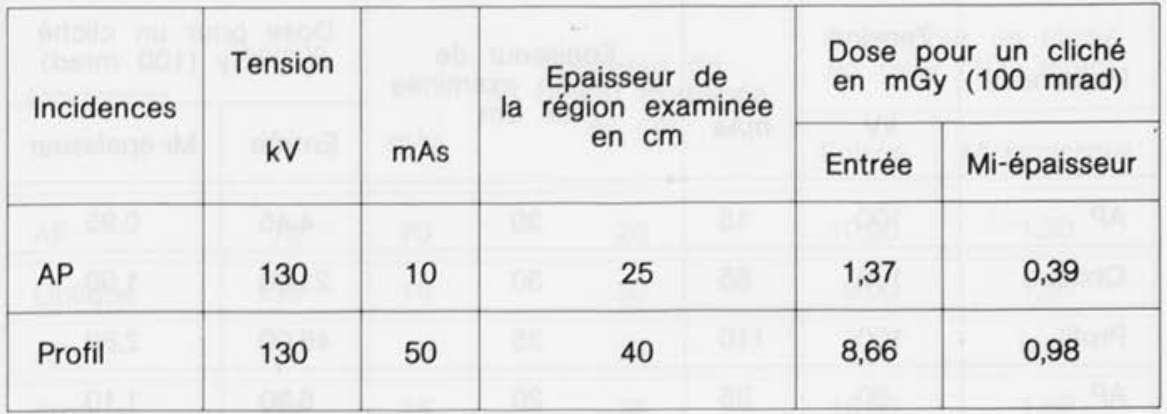

\section{ESTIMATION DE LA DOSE POUR L'ENSEMBLE D'UN EXAMEN TYPE}

- Pour un nombre moyen de clichés de 1,5

\begin{tabular}{|c|c|c|}
\hline \multicolumn{2}{|c|}{ Doses pour l'ensemble de l'examen (mGy) } \\
\hline Mi-épaisseur & Seins & Thyroïde \\
\hline 1,03 & 0,19 & 0,07 \\
\hline
\end{tabular}




\section{LAVEMENT BARYTE}

Format des films $36 \mathrm{~cm} \times 43 \mathrm{~cm}$ et $24 \mathrm{~cm} \times 30 \mathrm{~cm}$

Distance foyer-film $110 \mathrm{~cm}$

Type de générateur triphasé

Filtration totale $2,5 \mathrm{~mm} \mathrm{Al}$

\begin{tabular}{|c|c|c|c|c|c|}
\hline \multirow{2}{*}{ Incidences } & \multirow{2}{*}{$\begin{array}{c}\text { Tension } \\
\text { kV }\end{array}$} & \multirow[b]{2}{*}{ mAs } & \multirow{2}{*}{$\begin{array}{l}\text { Epaisseur de } \\
\text { la région examinée } \\
\text { en } \mathrm{cm}\end{array}$} & \multicolumn{2}{|c|}{$\begin{array}{l}\text { Dose pour un cliché } \\
\text { en mGy }(100 \mathrm{mrad})\end{array}$} \\
\hline & & & & Entrée & Mi-épaisseur \\
\hline AP & 100 & 15 & 20 & 4,45 & 0,95 \\
\hline Oblique & 100 & 55 & 30 & 21,00 & 1,90 \\
\hline Profil & 100 & 110 & 35 & 48,00 & 2,80 \\
\hline AP & 80 & 35 & 20 & 6,80 & 1,10 \\
\hline Oblique & 80 & 190 & 30 & 46,00 & 2,80 \\
\hline
\end{tabular}

\section{ESTIMATION DE LA DOSE POUR L'ENSEMBLE D'UN EXAMEN TYPE}

- Pour un temps de scopie de $170 \mathrm{~s}$

- Pour un nombre moyen de clichés de 9,5

\begin{tabular}{|c|c|c|c|c|c|}
\hline \multicolumn{5}{|c|}{ Doses pour l'ensemble de l'examen (mGy) } \\
\hline \multicolumn{2}{|c|}{ Intestin grêle } & \multicolumn{2}{c|}{ Ovaires } & \multicolumn{2}{c|}{ Testicules } \\
\hline HT $^{\star \star}$ & BT $^{\star \star \star}$ & HT & BT & HT & BT \\
\hline $22,00(2,00)^{\star}$ & $32,00(2,00)^{\star}$ & 4,30 & 6,10 & 2,10 & 3,10 \\
\hline
\end{tabular}

* Dose délivrée au cours du temps de scopie.

** Haute tension.

$\star \star \star$ Basse tension. 


\section{T.G.D.}

Format des films

Distance foyer-film

Type de générateur

Filtration totale

\begin{tabular}{|c|c|c|c|c|c|c|}
\hline \multirow{2}{*}{ Incidences } & \multirow{2}{*}{$\begin{array}{l}\text { Tension } \\
\text { kV }\end{array}$} & \multirow[b]{2}{*}{ mAs } & \multirow{2}{*}{$\begin{array}{c}\text { Epaisseur } \\
\text { la région exa } \\
\text { en cm }\end{array}$} & \multirow{2}{*}{$\begin{array}{l}\text { de } \\
\text { aminée }\end{array}$} & \multicolumn{2}{|c|}{$\begin{array}{l}\text { Dose pour un cliché } \\
\text { en mGy ( } 100 \text { mrad) }\end{array}$} \\
\hline & & & & & Entrée & Mi-épaisseur \\
\hline AP & 70 & 70 & 20 & & 10,00 & 1,30 \\
\hline Oblique & 130 & 15 & 30 & & 9,00 & 1,30 \\
\hline AP & 130 & 5 & 20 & & 2,20 & 0,65 \\
\hline Profil & 130 & 25 & 35 & & 18,00 & 1,80 \\
\hline
\end{tabular}

$36 \mathrm{~cm} \times 43 \mathrm{~cm}$ et $24 \mathrm{~cm} \times 30 \mathrm{~cm}$ $110 \mathrm{~cm}$ triphasé $2,5 \mathrm{~mm} \mathrm{Al}$

- Pour un temps de scopie de $150 \mathrm{~s}$

- Pour un nombre moyen de clichés de 9,6

\begin{tabular}{|c|c|c|c|c|c|}
\hline \multicolumn{5}{|c|}{ Doses pour l'ensemble de l'examen (mGy) } \\
\hline \multicolumn{2}{|c|}{ Duodénum } & \multicolumn{2}{c|}{ Ovaires } & \multicolumn{2}{c|}{ Testicules } \\
\hline$H^{\star \star}$ & BT & HT & BT & HT & BT \\
\hline $16,00(2,40)^{\star}$ & $36,00(2,40)^{\star}$ & 3,30 & 7,00 & 1,60 & 3,50 \\
\hline
\end{tabular}

* Dose délivrée au cours du temps de scopie.

** Haute tension.

*** Basse tension.

VOL. 23 - NUMERO SPÉCIAL 
5.9 .

\section{RACHIS LOMBO-SACRÉ}

Format des films $30 \mathrm{~cm} \times 40 \mathrm{~cm}$ et $24 \mathrm{~cm} \times 30 \mathrm{~cm}$

Distance foyer-film $110 \mathrm{~cm}$

Type de générateur triphasé

Filtration totale $2,5 \mathrm{~mm} \mathrm{Al}$

\begin{tabular}{|c|c|c|c|c|c|c|}
\hline \multirow[t]{2}{*}{ Incidences } & \multirow{2}{*}{$\begin{array}{c}\text { Tension } \\
\text { kV }\end{array}$} & \multirow{2}{*}{ mAs } & \multicolumn{2}{|c|}{$\begin{array}{l}\text { Epaisseur de } \\
\text { la région examinée } \\
\text { en cm }\end{array}$} & \multicolumn{2}{|c|}{$\begin{array}{l}\text { Dose pour un cliché } \\
\text { en mGy (100 mrad) }\end{array}$} \\
\hline & & & & & Entrée & Mi-épaisseur \\
\hline AP ou PA & 70 & 165 & 25 & 08 & 28,00 & 1,50 \\
\hline AP ou PA & 75 & 50 & 20 & at & 8,20 & 1,20 \\
\hline Profil & 80 & 80 & 25 & I & 17,50 & 1,80 \\
\hline Profil & 95 & 35 & 25 & . & 11,00 & 1,35 \\
\hline
\end{tabular}

ESTIMATION DE LA DOSE POUR L'ENSEMBLE D'UN EXAMEN TYPE

- Pour un temps de scopie de $80 \mathrm{~s}$

- Pour un nombre moyen de clichés de 4,4

\begin{tabular}{|c|c|c|}
\hline \multicolumn{2}{|c|}{ Doses pour l'ensemble de l'examen (mGy) } \\
\hline Corps vertébral-L5- & Ovaires & Testicules \\
\hline $10,50(1,10)^{\star}$ & $10,50(1,10)^{\star}$ & 0,70 \\
\hline
\end{tabular}

* Dose délivrée au cours du temps de scopie. 


\section{BASSIN}

Format des films

$36 \mathrm{~cm} \times 43 \mathrm{~cm}$ et $30 \mathrm{~cm} \times 40 \mathrm{~cm}$

Distance foyer-film $110 \mathrm{~cm}$

Type de générateur triphasé

Filtration totale

$2,5 \mathrm{~mm} \mathrm{Al}$

\begin{tabular}{|c|c|c|c|c|c|c|}
\hline \multirow{2}{*}{ Incidences } & \multirow{2}{*}{$\begin{array}{c}\text { Tension } \\
\text { kV }\end{array}$} & \multirow{2}{*}{ mAs } & \multirow{2}{*}{\multicolumn{2}{|c|}{$\begin{array}{l}\text { Epaisseur de } \\
\text { la région examinée } \\
\text { en } \mathrm{cm}\end{array}$}} & \multicolumn{2}{|c|}{$\begin{array}{l}\text { Dose pour un cliché } \\
\text { en mGy ( } 100 \text { mrad) }\end{array}$} \\
\hline & & & & & Entrée & Mi-épaisseur \\
\hline $\mathrm{AP}$ & 65 & 95 & 20 & 날. & 12,00 & 1,50 \\
\hline AP & 75 & 50 & 20 & 39 & 8,20 & 1,20 \\
\hline $\mathrm{AP}$ & 60 & 15 & 10 & & 1,50 & 0,50 \\
\hline
\end{tabular}

\section{ESTIMATION DE LA DOSE POUR L'ENSEMBLE D'UN EXAMEN TYPE}

- Pour un temps de scopie de $25 \mathrm{~s}$

- Pour un nombre moyen de clichés de 2,1

\begin{tabular}{|c|c|c|}
\hline \multicolumn{2}{|c|}{ Doses pour l'ensemble de l'examen (mGy) } \\
\hline Vessie & Ovaires & Testicules \\
\hline $3,60(0,40)^{\star}$ & $3,60(0,40)^{\star}$ & $17,50(1,90)^{\star}$ \\
\hline
\end{tabular}

* Dose délivrée au cours du temps de scopie. 


\section{A.S.P.}

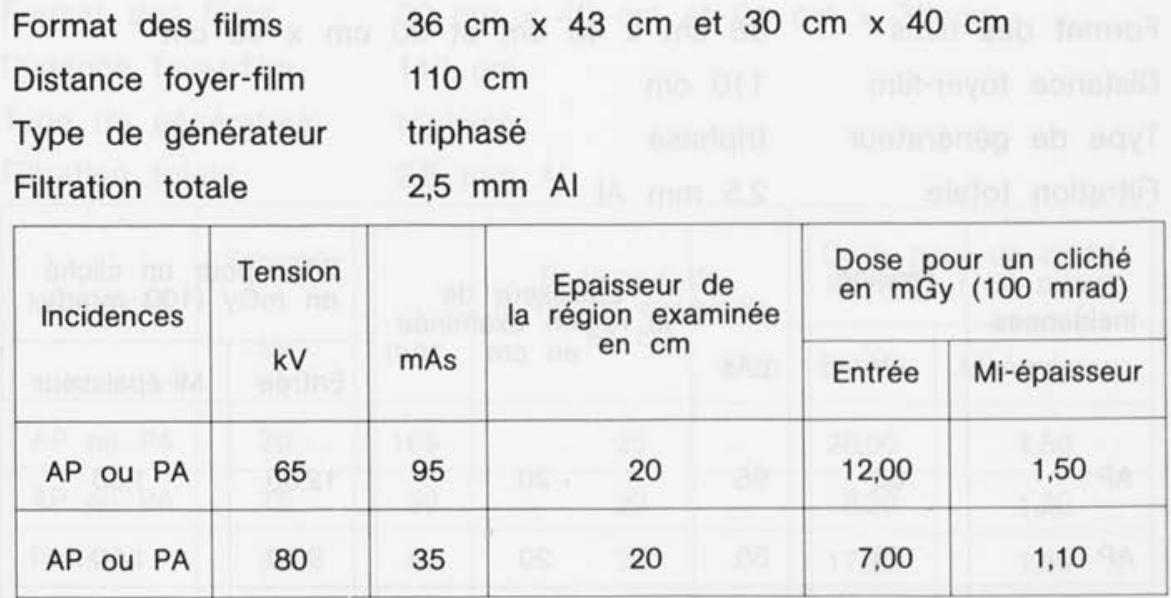

\section{ESTIMATION DE LA DOSE POUR L'ENSEMBLE D'UN EXAMEN TYPE}

- Pour un temps de scopie de $25 \mathrm{~s}$

- Pour un nombre moyen de clichés de 2,1

\begin{tabular}{|c|c|c|}
\hline \multicolumn{2}{|c|}{ Doses pour l'ensemble de l'examen (mGy) } \\
\hline Intestin grêle & Ovaires & Testicules \\
\hline $3,60(0,50)^{\star}$ & 3,50 & $<0,01$ \\
\hline
\end{tabular}

* Dose délivrée au cours du temps de scopie. 


\section{U.I.V.}

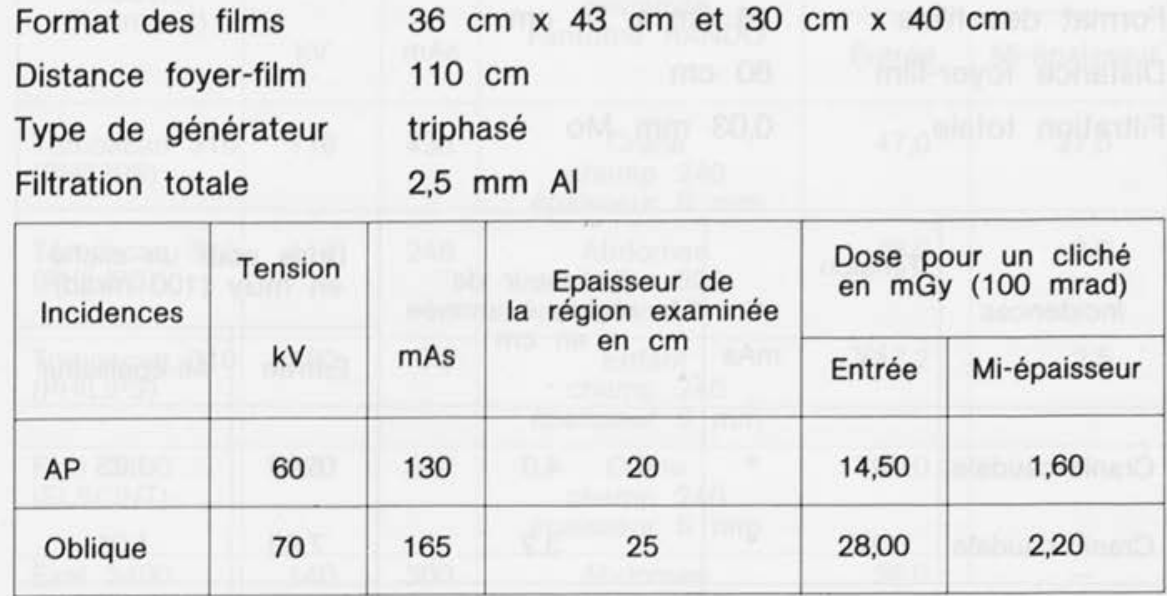

\section{ESTIMATION DE LA DOSE POUR L'ENSEMBLE D'UN EXAMEN TYPE}

- Pour un temps de scopie de $80 \mathrm{~s}$

- Pour un nombre moyen de clichés de 10,4

\begin{tabular}{|c|c|c|}
\hline \multicolumn{2}{|c|}{ Doses pour l'ensemble de l'examen (mGy) } \\
\hline Vessie & Ovaires & Testicules \\
\hline $19,50(1,10)^{*}$ & $19,50(1,10)^{*}$ & $70,00(3,95)^{\star}$ \\
\hline
\end{tabular}

* Dose délivrée au cours du temps de scopie. 


\section{MAMMOGRAPHIE}

Format des films

$18 \mathrm{~cm} \times 24 \mathrm{~cm}$

Distance foyer-film

$60 \mathrm{~cm}$

Filtration totale

$0,03 \mathrm{~mm} \mathrm{Mo}$

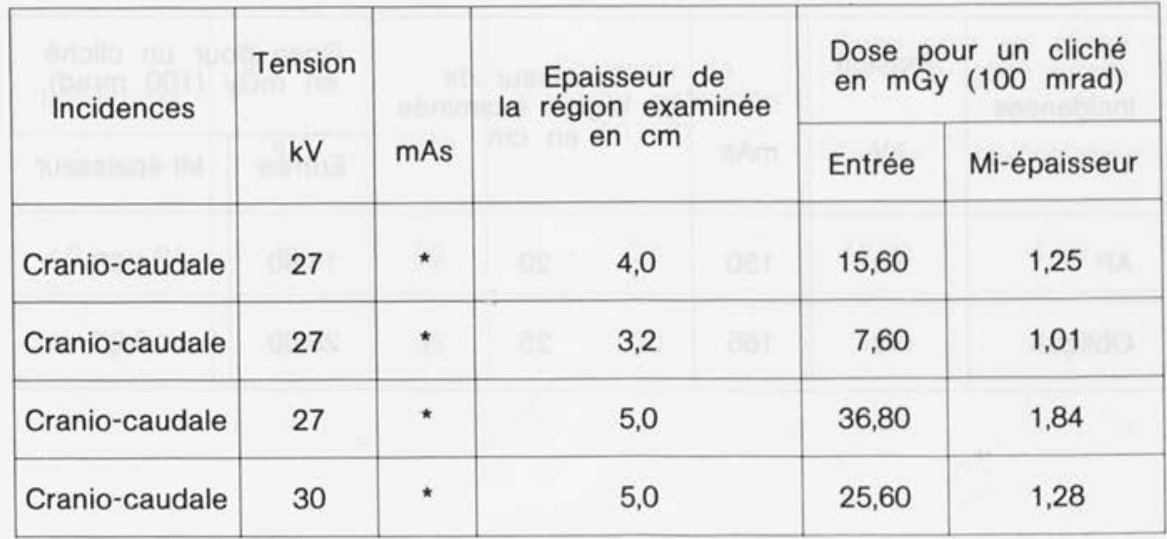

* Exposition automatique (cellule) 


\section{SCANOGRAPHIE}

A. Mesures utilisateurs

\begin{tabular}{|c|c|c|c|c|c|}
\hline \multirow{2}{*}{$\begin{array}{c}\text { Modèle } \\
\text { (Fabricant) }\end{array}$} & \multirow{2}{*}{$\begin{array}{c}\text { Tension } \\
\text { kV }\end{array}$} & \multirow[b]{2}{*}{$\mathrm{mAs}$} & \multirow{2}{*}{$\begin{array}{c}\text { Région } \\
\text { examinée } \\
\text { Fantôme RANDO }\end{array}$} & \multicolumn{2}{|c|}{$\begin{array}{l}\text { Dose pour une coupe } \\
\text { en mGy (100 mrad) }\end{array}$} \\
\hline & & & & Entrée & Mi-épaisseur \\
\hline $\begin{array}{l}\text { Tomoscan } 310 \\
\text { (PHILIPS) }\end{array}$ & 118 & 438 & $\begin{array}{c}\text { Crâne } \\
\text { champ } 240 \\
\text { épaisseur } 6 \mathrm{~mm}\end{array}$ & 47,0 & 27,0 \\
\hline $\begin{array}{l}\text { Tomoscan } 310 \\
\text { (PHILIPS) }\end{array}$ & 118 & 245 & $\begin{array}{c}\text { Abdomen } \\
\text { champ } 300 \\
\text { épaisseur } 12 \mathrm{~mm}\end{array}$ & 28,0 & 8,0 \\
\hline $\begin{array}{l}\text { Tomoscan } 310 \\
\text { (PHILIPS) }\end{array}$ & 100 & 72 & $\begin{array}{c}\text { Enfant } \\
\text { champ } 240 \\
\text { épaisseur } 6 \mathrm{~mm}\end{array}$ & 7,2 & 2,5 \\
\hline $\begin{array}{l}\text { Exel } 2400 \\
\text { (ELSCINT) }\end{array}$ & 120 & 300 & $\begin{array}{c}\text { Crâne } \\
\text { champ } 240 \\
\text { épaisseur } 5 \mathrm{~mm}\end{array}$ & 40,0 & - \\
\hline $\begin{array}{l}\text { Exel } 2400 \\
\text { (ELSCINT) }\end{array}$ & 140 & 300 & $\begin{array}{c}\text { Abdomen } \\
\text { champ } 420 \\
\text { épaisseur } 10 \mathrm{~mm}\end{array}$ & 36,0 & - \\
\hline $\begin{array}{l}\text { CE } 10000 \\
\text { (C.G.R.) }\end{array}$ & 130 & 578 & $\begin{array}{c}\text { Crâne } \\
\text { champ } 240 \\
\text { épaisseur } 5 \mathrm{~mm}\end{array}$ & 78,0 & 48,0 \\
\hline $\begin{array}{l}\text { CE } 10000 \\
\text { (C.G.R.) }\end{array}$ & 130 & 306 & $\begin{array}{c}\text { Abdomen } \\
\text { champ } 520 \\
\text { épaisseur } 10 \mathrm{~mm}\end{array}$ & 40,0 & 20,0 \\
\hline
\end{tabular}

B. Données constructeurs

\begin{tabular}{|l|l|c|c|}
\hline \multirow{2}{*}{ Fabricant } & \multirow{2}{*}{ Modèle } & \multicolumn{2}{c|}{ Dose pour une coupe en mGy } \\
\cline { 3 - 4 } & & Crâne & Corps \\
\hline ELSCINT & Exel 2400 & 27,0 & 24,0 \\
GENERAL ELECTRIC & GE 9800 & 25,0 à 30,0 & 25,0 à 30,0 \\
PHILIPS & Tomoscan TX & 60,0 & 24,0 \\
SIEMENS & Somatom DRH & 55,0 à 64,0 & 54,0 à 63,0 \\
THOMSON-C.G.R. & CE 12000 & 35,0 & 21,0 \\
\hline
\end{tabular}

VOL. 23 - NUMÉRO SPÉCIAL 


\section{BIBLIOGRAPHIE}

[1] FAGNANI F., THIERRY J.P., MACCIA C., LEFAURE C., BENEDITTINI M., MIZRAHI A., FAURE H. - Enquête nationale sur le radiodiagnostic - Aspects méthodologiques ( $1^{\text {re }}$ partie). J. Radiol., 1985, 66, 167-174.

[2] FAGNANI F., LEFAURE C., THIERRY J.P., MACCIA C., BENEDITTINI M., MIZRAHI A., FAURE H. - Enquête nationale sur le radiodiagnostic - Les moyens en personnel et en matériel ( $2^{\mathrm{e}}$ partie). J. Radiol., 1985, 66, 245-251.

[3] FAGNANI F., MACCIA C., LEFAURE C., BENEDITTINI M., MIZRAHI A., FAURE H. Enquête nationale sur le radiodiagnostic - Analyse comparative de l'activité des différents secteurs ( $3^{\mathrm{e}}$ partie). J. Radiol., 1985, 66, 329-336.

[4] COHEN G., WAGNER L.K., McDANIEL D.L., ROBINSON L.H. - Dose efficiency of screen-film systems used in pediatric radiography. Radiology, 1984, 152, 187193.

[5] HOLLOWAY A.F., CHENS C.N., PAIX D. - Speeds of films-screen combinations for radiography. J. Can. Assoc. Radiol. 1983, 34, 331-334.

[6] PICUS D., MCALISTER W.H., SMITH E., RODEWALD S., JOST R.G., EVEN R.G. Plain radiography with a rare-earth screen: comparison with calcium tungstate screen. $1984,143,1335-1338$.

[7] CHU W.K., SANGSTER W., DOBRY C.A. - Comparison of radiation exposure between conventional and rare-earth screen/film systems. Health Phys., 1985 , 49, $958-961$.

[8] Normes françaises C74.100. Appareils de radiologie, U.T.E.

[9] EDMONDS I.R. - Calculation of patient skin dose from diagnostic procedures. Bri. J. Radiol., 1984, 57, 733-734.

[10] SHRIMPTON P.C. - Calculation of patient skin dose from diagnostic X. ray procedures. Bri. J. Radiol., 1985, 58, 483-485.

[11] HARRISON R.M. - Backscatter factors for diagnostic radiology (HVL: 1-4 mm Al). Phys. Med. Biol., 1982, 27, 1465-1474.

[12] HARRISON R.M. - Tissue-air ratios and scatter-air ratios for diagnostic radiology (HVL: 1-4 mm Al). Phys. Med. Biol., 1983, 28, 1-18.

[13] Proceedings of a Scientific Seminar held in Udine, Italy, 17-19 april 1984. Criteria and Methods for quality Assurance in Medical X-ray Diagnosis. Bri. J. of Radiol., 1985, Suppl. $n^{\circ} 18$.

[14] BOUHNIK H., CHAVAUDA J. et Coll. - Commission Equipement groupe Diagnostic - France. Variabilité de la dose délivrée pour un cliché type sur un échantillon représentatif des installations radiologiques en France: extension de l'enquête de la SFPH, XXIII ${ }^{e}$ CONGRES SFPH, Nice, 1984.

[15] COSTA A., GABILLAT J.M., AUBANEL D. - Dosimétrie d'une installation de mammographie. XXIII ${ }^{e}$ Congrès SFPH, Nice, 1984.

[16] BOUHNIK H. - Paramètres agissant sur la qualité de l'image en mammographie. Cours de mammographie sous la direction de A. LE TREUT, Bordeaux, 1984. 
[17] ROSENWALD J.C. - Irradiation du sein lors des examens mammographiques. Cours de mammographie sous la direction de A. LE TREUT, Bordeaux, 1984.

[18] NOËL A., STINES J., ALETTI P., BEY P., HOEFFEL J.C. - Mesure de la dose délivrée au cours des examens scanographiques. XXIII ${ }^{\mathrm{e}}$ Congrès SFPH, Nice, 1984.

[19] AUBERT B., BRIDIER A. - Caractéristiques et performances du scanographe CE 10000 testées pendant trente mois d'utilisation. Journal d'Imagerie Médicale, $1983,1,37-45$.

[20] AUBERT B. - Mesures de la dose en radiodiagnostic et tomodensitométrie. Journal de Radiologie, 1981, 62, 587-589.

\section{OUVRAGES DE RÉFÉRENCE}

- Traité de radiodiagnostic - 1 . L'image radiologique.

J. DUTREIX, V. BISMUTH, M. LAVAL-JEANTET. Masson et Cie.

- Graphs and tables for use in Radiology.

F. WACHSMANN, G. DREXLER. Springer-Verlag, Berlin, Heidelberg, New York.

- The calculation of dose from external photon exposures using references human phantoms and Monte Carlo methods - Part III: Organ doses in X-rays diagnosis. GSF-Bericht S-1026, 1984.

G. DREXLER, W. PANZER, L. WIDENMANN.

- Quality assurance in Diagnostic Radiology. World Health Organization (1982).

- International Commission on Radiological Protection. Protection of the patient in Diagnostic Radiology. ICRP Report N $N^{\circ} 34$ (1982).

- Measurement of the Performance Characteristic of Diagnostic X-Ray Systems Used in Medecine. Part III: Computed Tomography X-Ray Scanners. The Hospital Physicist's Association - Topic Group Report 32.

- National Council on Radiation Protection and Measurements. Mammography - A user's Guide. NCRP Report No 85 (1986). 